\title{
Interface Design and Evaluation for CAS Systems
}

\author{
Cristiano Paggetti ${ }^{1}$, Sandra Martelli ${ }^{2}$, Laura Nofrini ${ }^{2}$, Paolo Vendruscolo ${ }^{2}$ \\ ${ }^{1}$ MEDEA - MEDical and Engineering Applications, Firenze, Italy \\ c.paggetti@medea-italia.it \\ ${ }^{2}$ Biomechanics Lab, Istituti Ortopedici Rizzoli, Bologna, Italy \\ \{S.Martelli, L.Nofrini, P.Vendruscolo\}@biomec.ior.it \\ http://www.ior.it/biomec/
}

\begin{abstract}
The use of Computer Assisted Surgery (CAS) systems is becoming very common in the clinical practice, therefore the evaluation of such systems in terms of clinical outcomes and ergonomic features is more and more relevant. This paper goals has been to define some domain specific guidelines for the design of Human Computer Interfaces (HCI) for surgical application and to provide an evaluation protocol of existing CAS systems. The demonstration application has been a planning system developed for the Total Knee Replacement (TKR), a high skill demanding procedure, where the planning phase is crucial for the success of the intervention. The results we have obtained can be extended also to surgical training systems and surgical navigation platforms.
\end{abstract}

\section{Introduction}

The use of Computer Assisted Surgery (CAS) systems is becoming very common in the clinical practice, therefore the evaluation of such systems in terms of clinical outcomes and ergonomic features is more and more relevant. This paper addresses the definition of some domain specific guidelines for the design of Human Computer Interfaces (HCI) for surgical application. Currently the literature does not provide much indications related to the HCI in such a framework [7] [8]. Starting from the application problem analysis and the user requirements, this paper explains how the system specifications have been implemented and which criteria have been applied for the evaluation of the HCI. The paper's goal is therefore to provide a consistent approach for the HCI design and an evaluation protocol of existing CAS systems. The domain addressed is related to the planning systems and it is suitable also for training and surgical navigation ones.

In this paper the demonstration application is the Total Knee Replacement (TKR), a high skill demanding procedure, where the planning phase is crucial for the success of the intervention. The aim of TKR is to restore the correct alignment of the mechanical axis of the limb and the correct function of the knee joint. In particular the HCI discussed in this paper assists the surgeon in the planning of correct size, orientation and position of the prosthesis components for TKR interventions. 


\section{Material and Methods}

\subsection{HCI Design}

We recall that the main objectives of most Computer Assisted Systems (CAS) are[1][2]:

i. enhance surgeon's 3D perception of the surgical scenario;

ii. enhance surgeon's dexterity in performing high skill demanding actions;

iii. enhance the accuracy and repeatability of surgeon's action;

iv. reduce the invasiveness of the surgical intervention;

v. reduce the intraoperative decision time.

The universal access to a HCI to use CAS systems implies to address general requirements during its design, with a systematic approach related to the functionality implementation as well as the features of the specific application domain.

We suggest the following methodological approach:

1. identification of the users and of the working conditions

2. definition of the system's output (i.e. goals and specific outcomes)

3. definition of the system's input

4. definition of the system functionalities to treat input data to obtain required output

5. identification of the logical phases to subdivide and organize the overall procedure

6. definition of the user's interaction modality

We underline that the HCI design, although usually proposed by a scientist, needs a constant involvement of the users' in the selection of data, display layout and also testing

\subsection{Evaluation Protocol for CAS HCI}

The evaluation of CAS HCI was performed during repeated tests of the planning procedure. Trials were conducted on 10 patients by four surgeons (two expert surgeons and two junior surgeons), who planned the intervention on all patients; one of them repeated planning four times in different days.

In particular, it has been worked out an evaluation based on the analysis of the user's reactions as respect to the HCI implementation. Comments and reactions have been divided in two groups, those related to features correctly implemented in the CAS system and those features that can be suggested in order to improve the usability and the user's satisfaction.

Furthermore the outcomes of the questionnaire, based on the Guidelines for designing user interface software[9] from the MITRE Corporation, are discussed directly with the programmer and the HCI expert. It is worthwhile to carry out the evaluation without analysing the results of the surgeons involved in the HCI design.

The CAS HCI evaluation was based on three different aspects, originally defined by the authors: 
1. evaluation of the user's satisfaction in using HCI, through interviews to the users and a specific questionnaire

2. evaluation of the user's ability and fatigue in using the HCI, through objective observation of the system's behavior by an independent scientist

3. evaluation of the planning repeatability as an index of the HCI efficacy.

The first item concerns the subjective feeling of the surgeons in using the system's tools: friendliness, ease of use, lack of tools that could be useful to successfully reach the system's aims.

The second item concerns the evaluation of objective aspects of the system: time necessary to complete a particular action, the implemented data control, errors' management, workspace layout.

The third item concerns quantitative evaluations of the system performances.

\section{Results}

\subsection{Implementation of HCI Design}

The six methodological criteria reported in $\$ 2.1$ were applied to the TKR planning HCI design, with the following results:

1. users are orthopaedic surgeons working in a pre-operative framework;

2. the system's output is the prosthesis components location with respect to the patient anatomy;

3. the system's input are patient CT data and CAD drawings of different prosthesis,

4. system functionality include patient selection, anatomical marker selection, virtual prosthesis positioning,

5. the TKR planning can be divided in two phases: the Alignment phase and the Positioning phase

6. the user's interaction modality is mouse based; in particular the selection criteria is based on a "one click" approach, simple menus to deal with input data, data elaboration and on-line help.

In practice the TKR planner consists of two successive steps and corresponding screen: the Alignment phase and the Positioning phase.

In the first, the frontal and the lateral scouts of the limb are shown together with a well distiguished command menu with sorted buttons corresponding to the expected user's actions (Fig.1). The surgeon identifies the 'ideal' limb mechanical axis in the two scout views (the line connecting the hip center and the ankle center passing through the knee center), adjusting three geometrical frame-markers (a circle for the ankle center a cross for the knee center and a rectangle for the ankle center) to the patient's anatomy by drag and drop with the mouse. Two buttons, called UNDO and REDO, allow the surgeon to move across the ten latest performed actions. Moreover there is a DATA button that provides the surgeon with the information about the realignment angle: this is computed on the frontal scout view of the patient limb and 
is the $2 \mathrm{D}$ angle between the line joining the hip center and the center of the distal part of the femur and the line between the proximal tibia center and the ankle center.

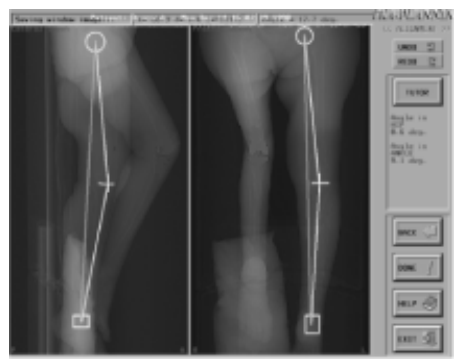

Fig. 1. Alignment phase

In the second planning step, the positioning phase (Fig.2), the surgeon defines the orientation, placement and size of the two prosthetic components. In this phase, the user interface includes four data windows: the frontal and the lateral projection of the joint (X-rays like image computed from CT data), a window displaying sections of the bone computed in any user's desired position and orientation and a windows with the $3 \mathrm{D}$ reconstruction of the joint.

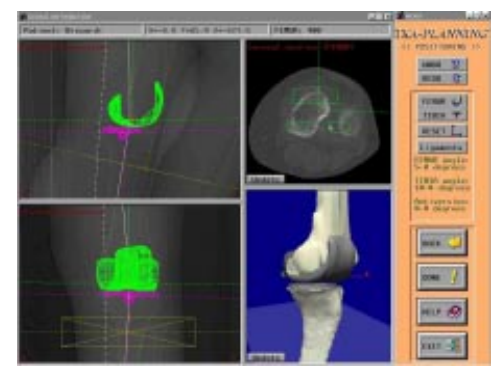

Fig. 2. Positioning phase

During the positioning phase, the arrangement of the components can be verified and interactively adjusted by the surgeon using the system tools: he can drag the prosthesis with the mouse onto a frontal projection, lateral and arbitrary oriented section planes of the knee joint. All the images can be zoomed and the system provides the surgeon with some $3 \mathrm{D}$ measures, such as current correction angle, that help the surgeon to verify if the current one is the desired result.

Moreover a command menu is present on the screen, with a position, layout and organization similar to that of the previous phase. It includes buttons similar to the previous ones and specific command buttons: UNDO and REDO, whose task is the same as for the alignment phase, RESET, that allows the system to restore the initial state, FEMUR and TIBIA that are the two buttons to enter the two prosthesis databases. These databases includes the prosthesis templates of different sizes both for the femur and for the tibia. 


\subsection{Evaluation of the Implemented CAS HCI}

The evaluation was performed recording users' comments and suggestions and by means of a questionnaire based interview. The main outcomes have been summarized in three groups, one for each aspect described in $\$ 2.2$.

As concerns the user's satisfaction about the system, the surgeons expressed positive judgements on data issuing methods: patients' data are loaded only one time at the beginning of the planning session, choosing the patient directly from a list. During all the planning session the system shows both patient's CT images and information (name, leg on which is planned to intervene) derived directly from patient's CT slice header; this way the surgeon is guaranteed that the patient is the right one, without any effort.

About issuing methods during the interaction with the system, the most appreciated aspect is that data are issued according only through the mouse, avoiding the shifting from the board to the mouse and back.

Other aspects that have been appreciated are that the visualisation of the areas used to issue data are sufficiently clear, even because the surgeon can easily zoom the images' areas he is interested in, and the fact that the data issuing rhythm is defined by the user and not imposed by the system: the surgeon can take as much time as he needs to fulfil any task.

Moreover the user's actions and the system's reactions are similar as concerns similar and always predictable actions and the set of words used to control the operations' sequence is always coherent.

Finally the transactions' sequence is logic from the user's point of view, reflecting the steps of the surgical technique.

In order to increase the software usability, users have suggested to introduce the visualization of the scale according which values are represented and what they refers to (for instance, angular values are visualized, but is not clear what they are referring to). Moreover, it could also be useful to visualize within a defined area of the screen an hint concerning data to be issued in order to fulfill the planning phase.

The prosthesis and the tools in general should be represented with the least number of particulars, so that the references on the underneath $\mathrm{CT}$ image remains as much as possible visible.

Finally the surgeons would be pleased to have a results' preview, to be sure of his work be correct.

Among the valuations done by an independent scientist in order to assess user's fatigue in using HCI, noteworthy is the fact that windows are never superimposed and the working area is very well organized, with all the information and buttons on the left of the screen and the patient's images on the other side. Buttons are labeled with self explanatory commands and correspondent buttons remains the same in all the phases (for instance, the EXIT button appears in all the phases, always in the same position)

Moreover has been appreciated the fact that there is always an immediate feedback between what appears on the screen and the issued or the given command: the system does not suffer delays in detecting the position of the cursor, the cursor itself is 
always stable and visible, and when time needed at the end of the reckoning it is correctly shown through an indicator.

The lines tracking avails itself of the elastic effect, which allows to see immediately how the traced line will come out.

Further aspects positively valued have been that data can be cancelled or modified only by the user(only in one particular situation the user can delete data without being informed; we will describe this situation among the features that have to be included in the system in order to improve the usability), and that data processing starts always explicitly: the computer does never take the control automatically and in case of error's messages the program's execution is not interrupted.

Finally there is no chance of messing the system's messages and helps although the system never overload the user with too much information.

In order to improve the system's usability and to reduce the user's fatigue, some features have been suggested to be introduced, like a messages asking if data are required to be cancelled whenever destructive actions are carried (RESET) or asking if it is really required to exit and save the configuration currently on at the exit from the operative phase. Moreover it should be possible to save the intermediate data and eventually to go back automatically to the saved configuration.

Another feature that should be introduced is to unable buttons and tools when these are not usable.

Finally the cursor should vary the shape according to the area which is in. The use of the arrow cursor is not the best one in a graphic work area (a cross should probably be better).

Testing results concerning the planning repeatability are shown in Table 1 .

\begin{tabular}{|c|c|}
\hline TKA Computer Planning & \\
\hline Accuracy of measures done on scout images $( \pm 1$ pixel) & $\mathbf{0 . 6} \mathbf{~ m m}$ \\
\hline Accuracy of measures done on images derived from slices $( \pm 1$ pixel) & $\mathbf{0 . 3} \mathbf{~ m m}$ \\
\hline $\begin{array}{l}\text { Repeatability of interactive anatomical choices (anatomical points } \\
\text { identification) }\end{array}$ & $\mathbf{3 . 8} \mathbf{~ m m}$ \\
\hline $\begin{array}{c}\text { Repeatability of interactive anatomical choices (long mechanical axis } \\
\text { identification) }\end{array}$ & $\mathbf{1}^{\circ}$ \\
\hline System's reliability (anatomical points identification) & $\mathbf{6 . 2} \mathbf{~ m m}$ \\
\hline System's reliability (mechanical axis identification) & $\mathbf{1 . 2}^{\circ}$ \\
\hline
\end{tabular}

Table 1- Testing results

\section{Discussion}

It is difficult to evaluate if the proposed approach is "optimal", but we just observe that the general user's feeling is easy of use and confidence in the outcome (22 over 27.75 , about $80 \%$ ), that the use of the HCI is consistent and focused on the most important parameters. In fact the planner outcomes are very repeatable especially in the significant final variables, i.e. the mechanical axis (variability of $1^{\circ}$ for the same 
surgeon and $1.2^{\circ}$ for different surgeons). These data are the most important in Table 1, because determine the automatic prosthesis initial placement; the results show that the indication for the limb realignment guarantees quantitative reliable results.

Another important and original aspect of this paper is the proposed evaluation method. It is a new method, only partially inspired to standard techniques [3] [4] [5] [6] and absent in the previous descriptions of CAS system and also TKR planning[10] [11][13] [14].

We have proposed a detailed description of the main issues in the evaluation procedure $(\$ 2.2)$ and verified that in our TKR planning software they were able to provide useful indications of the HCI efficacy and also on further developments of its design and functionality. In particular the user's indications and the objective observation have allowed to correct mainly the user's interaction modality, increase the number of information that the user can easily handle, and this result confirm the impression that even a careful and systematic method to HCI design should be always followed by a similar careful and systematic testing phase, able to optimize the specific software outcome.

We remark that the methodological guidelines and most of the specific observations can be useful to other groups developing or testing surgical planners(especially in orthopaedics).

Moreover we underline that also surgical training systems and surgical navigation platforms share a similar data treatment and HCI layout, because the final goal is still an image guided definition of the optimal surgical strategy(preoperatively in our case, in simulation modality in training systems, intraoperatively in navigation platforms).

Therefore these kind of applications can benefit directly from the proposed approach to HCI design and evaluation. However this approach cannot yet be extended simply to registration or execution HCI for robotic or automatic systems, but just represent a first step toward a standardized procedure to design efficient and safe $\mathrm{HCI}$ in CAS systems.

\section{References}

1 "Computer Assisted Orthopedic Surgery (CAOS)", by L.P. Nolte and R. Ganz (eds.), Hogrefe Huber Publishers, Seattle-Toronto-Bern, 1999, ISBN 0-88937-168-7.

2. Taylor, R.H., S. Lavalée. Computer Integrated Surgery. Cambridge: MIT Press, 1996. Clinical Orthopaedics and Related Research, Number 354 September 1998 (Special issue).

Patkin M., Isabel L., "Ergonomics, engineering \& surgery of endo_dissection" J. R. Coll. Surg. Edinb., 40 -April 1995.

3. Sater-Black K., Iversen N. "How to conduct a design review" Mechanical Eng., 89-92, March 1994

4. Picard F., Tourne Y., Saragaglia D. "Computer Assisted Knee Replacement/Ergonomic Evaluation" Proceedings of the Millennium Symposium on Computer Assisted Orthopaedics Surgery(CAOS 2000), Davos (Switzerland), February 17-19, 2000.

5. Zimolong a., Radermacher K., Mengel M., Friedrichs D., Rau G. "User-Interface and InputDevices for the CRIGOS Surgical Robot" Proceedings of the Millennium Symposium on 
Computer Assisted Orthopaedics Surgery(CAOS 2000), Davos (Switzerland), February 1719, 2000.

6. Vendruscolo P., Martelli s., "Interfaces for computer and robot assisted surgical systems", Information and Software Technology, Vol. 43, N. 2, pp. 87 - 96, 2001 (Feb)

7. Ollson E., Boralv E., Goransson B., Sandblad B., "Domain specific style guides - design and implementation" Proceedings of the Motif and COSE International User Conference, Washington DC, 133 - 139 (1993).

8. Ameritech Standard for information systems, Ameritech services, 1993, 1994, 1995, 1996.

9. Smith S.L., Mosier J.N., "Guidelines for designing user interface software", the MITRE Corporation, 1986.

10. Fadda M., Bertelli D., Martelli S., Marcacci M., Dario P., Paggetti C., Caramella D., Trippi D.: "Computer assisted planning for total knee arthroplasty". In "Lecture Notes in Computer Science: CVRMed - MRCAS'97", Vol. 1205, Troccaz, Grimson and Mösges Eds., Springer (ISBN 3-540-62734), 619-28, 1997.

11. Delp S.L., Stulberg S.D., Davies B., Picard F., Leiter F. "Computer Assisted Knee Replacement”. Clin Ort. 354:51-56, 1998.

12. Martelli S., Marcacci M. et al. "Computer and robot assisted total knee replacement: analysis of a new surgical procedure". Annals of biomedical engineering Vol. 28 1-8, 2000

13. Muller W., Bockholt U., Voss G., Lahmer A., Borner M., "Planning System for Computer Assisted Total Knee Replacement" In Medicine Meets Virtual Reality- J.D. Westwood et al. (eds) IOS Press 214-219, 2000.

14. Wolsiffer K., Kalender W.A. "A feasibility study on computer assisted planning of total knee replacement surgery" Proceedings of the Millennium Symposium on Computer Assisted Orthopaedics Surgery(CAOS 2000), Davos (Switzerland), February 17-19, 2000. 